\title{
Adição da macroalga Kappaphycus alvarezii em rações de frangos de corte na fase inicial
}

\author{
Feed incorporated with the macroalgae Kappaphycus alvarezii for broiler chickens in the \\ starter phase
}

Sônia Maria de Brito Marques Quirino ${ }^{1}$, Débora Vaccari Quaresma ${ }^{2}$, Felipe Dilelis ${ }^{2 \star}$, Leonardo Willian de Freitas ${ }^{3}$, Cleriston Andrade Machado ${ }^{2}$, Eduardo Vellozo ${ }^{2}$, Marcos Fabio de Lima ${ }^{4}$, Cristina Amorim Ribeiro de Lima ${ }^{2}$

\author{
${ }^{1}$ Colégio Técnico da Universidade Federal Rural do Rio de Janeiro, Seropédica, RJ, Brasil. \\ ${ }^{2}$ Universidade Federal Rural do Rio de Janeiro, Seropédica, RJ, Brasil. Autor para correspondência: fdilelis@ufrrj.br \\ ${ }^{3}$ Universidade Federal do Mato Grosso, Sinop, MT, Brasil. \\ ${ }^{4}$ Instituto Federal do Rio de Janeiro, Pinheiral, RJ, Brasil.
}

Submissão: 20/03/2020 / Aceite: 02/09/2020

\begin{abstract}
RESUMO
Foi realizado um experimento para avaliar a digestibilidade de nutrientes, o desempenho zootécnico e parâmetros de qualidade óssea de frangos de corte alimentados, de um a 21 dias de idade, com rações contendo níveis crescentes da macroalga Kappaphycus alvarezii. $O$ total de 150 frangos da linhagem Cobb $500^{\circledR}$ foram distribuídos em um delineamento experimental inteiramente casualizado, com cinco tratamentos, seis repetições e cinco aves por repetição. O ensaio de metabolismo utilizado foi o de coleta total de excretas, do $15^{\circ}$ ao $20^{\circ}$ dia. Os parâmetros ósseos avaliados foram o índice de Seedor, resistência a quebra e cinzas ósseas. A macroalga seca e moída foi adicionada às rações de frango de corte nos valores de $0 \%, 0,5 \%, 1,0 \%, 1,5 \%$ e 2,0\%; em substituição ao inerte caulim. A adição de Kappaphycus alvarezii não influenciou os parâmetros de desempenho, os parâmetros de qualidade óssea e os coeficientes de retenção de cinzas e do fósforo. Na avaliação da digestibilidade das rações foram observadas reduções $(p<0,05)$ na energia metabolizável aparente e nos coeficientes de digestibilidade da matéria seca, da proteína bruta e da energia bruta com a inclusão da alga. A alga Kappaphycus alvarezii apresenta potencial como aditivo nas rações de frangos de corte, mas deve ser considerada a possível diminuição da digestibilidade da dieta.
\end{abstract}

PALAVRAS-CHAVE: alga, aminoácidos, desempenho, digestibilidade, qualidade óssea.

\section{ABSTRACT}

An experiment was conducted to evaluate the nutrient digestibility, zootechnical performance, and bone quality parameters of broilers fed a diet containing increasing levels of the macroalgae Kappaphycus alvarezii from one to 21 days of age. A total of 150 Cobb $500^{\oplus}$ broilers were distributed in a completely randomized design with five treatments, six replications, and five birds per replicate. The total excreta collection method was applied from the $15^{\text {th }}$ and $20^{\text {th }}$ day for the metabolism trial. The following bone quality parameters were assessed: the Seedor index, bone strength, and bone ashes. Dry ground macroalgae were added to the feed in the levels $0 \%, 0.5 \%, 1.0 \%, 1.5 \%$ and $2.0 \%$; replacing the inert kaolin. Incorporation of Kappaphycus alvarezii did not influence performance parameters, bone quality parameters, or the coefficients of retention for bone ash and phosphorus. As for feed digestibility, decreases $(p<0.05)$ were observed in apparent metabolizable energy and the coefficients of digestibility for dry matter, crude protein, and crude energy with the inclusion of algae. The algae Kappaphycus alvarezii shows potential as an additive in broiler feed, but a possible decrease in digestibility deserves consideration.

KEYWORDS: algae, amino acids, performance, digestibility, bone quality.

\section{INTRODUÇÃO}

As macroalgas marinhas são organismos multicelulares classificados em três grandes grupos de acordo com a coloração do talo: algas marrons (Phaeophyta), algas vermelhas (Rhodophyta) e algas verdes 
(Chlorophyta) (EL GAMAL 2012). A utilização de macroalgas como ingredientes na alimentação animal tem sido estudada por diversos grupos de pesquisa (MELO et al. 2008, ABUDABOS et al. 2013), com resultados positivos quanto a sua inclusão em dietas para aves. Ações anti-inflamatória, prebiótica e antimicrobiana têm sido relatadas devido à presença de compostos fitoquímicos presentes nessas algas (CORNISH \& GARBARY 2010, IJI \& KADAM 2013, PUSHPARAJ et al. 2014).

A Kappaphycus alvarezii é uma macroalga vermelha (Rhodophyta), cultivada em diversos países tropicais como fonte de matéria prima para a produção de kapa carragena (polissacarídeo sulfatado), usado como agente estabilizante, gelatinizante, espessante e emulsificante pelas indústrias de alimentos, farmacêutica e cosmética. Sua composição centesimal varia de acordo com o local e condições de cultivo (KUMAR et al. 2015, XIREN \& AMINAH 2017), sendo de 4,5\% a $12 \%$ de proteína bruta; $0,4 \%$ a $1 \%$ de extrato etéreo; $16 \%$ a $33 \%$ de cinzas e de $7 \%$ a $18 \%$ de fibra bruta na matéria seca. A presença de diferentes compostos fitoquímicos foi constatada nesta macroalga, dentre eles: alcaloides, taninos, terpenóides, flavonoides, saponinas e compostos fenólicos (PRABHA et al. 2013).

O cultivo da Kappaphycus alvarezii se encontra bem estabelecido em diversos países, destacando-se a Indonésia e Filipinas (FAO 2012) e a produção atingiu valores acima de oito milhões de toneladas de biomassa úmida, liderando o ranking das algas mais produzidas mundialmente. Apesar de ser uma das algas mais cultivadas no mundo, poucos trabalhos estão disponíveis sobre a inclusão de Kappaphycus alvarezii como ingrediente em rações de frangos de corte, sendo necessários trabalhos que avaliem a sua inclusão, bem como a composição nutricional.

Objetivou-se com este trabalho avaliar níveis de inclusão da macroalga em dietas para frangos de corte de um a 21 dias de idade sobre a digestibilidade de nutrientes, o desempenho zootécnico e a qualidade óssea das aves.

\section{MATERIAL E MÉTODOS}

Os procedimentos adotados neste ensaio foram aprovados pelo Comitê de Ética no Uso de Animais CEUA/IZ/UFRRJ (Processo no 23083.000039/2018-28).

A macroalga utilizada foi produzida na llha Grande, litoral sul do estado do Rio de Janeiro, Brasil. A alga foi recebida da forma como é destinada à indústria, seca ao sol, após lavagem na própria água do mar. O material foi então triturado em moinho de facas em peneira de $2 \mathrm{~mm}$ de abertura para posterior fabricação das dietas experimentais.

Uma alíquota do material foi moída em micromoinho de facas tipo Willey com peneira de $1 \mathrm{~mm}$, para análises laboratoriais. Foram determinadas a matéria seca (MS), energia bruta (EB), proteína bruta (PB), extrato etéreo $(E E)$, fibra bruta $(F B)$, matéria mineral $(M M)$, cálcio $(\mathrm{Ca})$ e fósforo $(P)$ de acordo com $A O A C$ (1995). Fibra em detergente neutro (FDN) e fibra em detergente ácido (FDA) de acordo com VAN SOEST et al. (1991) e minerais (INSTITUTO ADOLF LUTZ 2008). As análises de ácidos graxos e aminoácidos foram realizadas em laboratório comercial (CBO Análises Laboratorias, SP, Brasil). A composição da Kappaphycus alvarezii utilizada no ensaio está descrita na Tabela 1.

Foram utilizados 150 pintos machos de um dia de idade, Cobb $500^{\circledR}$. As aves foram vacinadas no incubatório contra as doenças de Gumboro, Marek e Bouba aviária, sendo vacinadas contra New Casttle aos 10 dias de idade, através da via ocular. Ao serem alojados, os pintos foram pesados e então distribuídos nas gaiolas metabólicas de acordo com o peso médio $(37 \mathrm{~g} \pm 1,92)$.

Foram utilizadas baterias metálicas de três andares, sendo cada andar subdividido em quatro compartimentos, totalizando 30 gaiolas, cada gaiola com medidas de $0,90 \times 0,40 \times 0,40 \mathrm{~m}$, contendo bebedouro automático tipo copo e comedouro tipo calha e com sistema de aquecimento por lâmpadas incandescentes. As temperaturas, máxima média e mínima média, foram de $29{ }^{\circ} \mathrm{C}$ e $24{ }^{\circ} \mathrm{C}$, respectivamente. $O$ fornecimento de luz foi de 23 horas nos primeiros 10 dias, com redução para 16 horas de luz e oito horas de escuro após essa idade. Água e ração foram fornecidas ad libitum.

O delineamento experimental utilizado foi o inteiramente casualizado (DIC), com cinco tratamentos (valores de inclusão da macroalga), seis repetições por tratamento e cinco aves por unidade experimental.

Os tratamentos foram estabelecidos a partir da ração referência (Tabela 2) que foi suplementada com valores crescentes da macroalga em substituição ao inerte (caulim). Os tratamentos foram 0;0,5; 1,0;1,5 e $2,0 \%$ de inclusão da alga Kappaphycus alvarezii, totalizando cinco rações experimentais, em uma única fase (1 a 21 dias de idade). A ração referência foi formulada de forma a atender no mínimo as exigências nutricionais para frangos de corte preconizadas por ROSTAGNO et al. (2017).

O período experimental foi do $1^{\text {o }}$ ao $21^{\circ}$ dia de idade das aves. O fornecimento de ração foi mensurado durante todo o período experimental, para determinação do consumo de ração. Aos 21 dias de 
idade as aves foram pesadas, para determinação: do peso vivo, ganho de peso e conversão alimentar. A mortalidade foi monitorada e registrada diariamente.

Tabela 1. Composição centesimal, ácidos graxos, aminoácidos e microminerais da Kappaphycus alvarezii obtida da região do litoral sul-fluminense ${ }^{1}$.

Table 1. Proximate composition, fatty acids, amino acids, and microminerals of Kappaphycus alvarezii obtained from the southern coast region of the state of Rio de Janeiro ${ }^{1}$.

\begin{tabular}{|c|c|c|c|c|c|}
\hline Composição centesima & & Bas & $\mid \mathrm{ral}^{1}$ & Bas & \\
\hline Matéria seca (\%) & & & & & \\
\hline Proteína bruta (\%) & & & & & \\
\hline Extrato etéreo (\%) & & & & & \\
\hline Energia bruta (kcal/kg) & & & & 231 & \\
\hline Matéria mineral (\%) & & & & & \\
\hline Fibra em detergente ne & & & & & \\
\hline Fibra em detergente áci & & & & & \\
\hline Cálcio (\%) & & & & & \\
\hline Fósforo (\%) & & & & & \\
\hline Sódio (\%) & & & & & \\
\hline Potássio (\%) & & & & & \\
\hline Ácidos graxos $^{1}$ & $\%$ & Aminoácidos $^{1}$ & $\%$ & Microminerais & $\mathrm{mg} / \mathrm{kg}$ \\
\hline Ácido merístico & 0,03 & Ácido aspártico & 0,13 & Ferro & 782 \\
\hline Ácido meristoleico & 0,01 & Ácido glutâmico & 0,25 & Cobre & 3,1 \\
\hline Ácido pentadecanoico & 0,01 & Serina & 0,14 & Zinco & 21,2 \\
\hline Ácido palmítico & 0,52 & Glicina & 0,18 & Manganês & 9,3 \\
\hline Ácido palmitoleico & 0,04 & Histidina & 0,02 & Cádmio & 0,9 \\
\hline Ácido marárico & 0,01 & Arginina & 0,13 & Chumbo & 1,8 \\
\hline Ácido esteárico & 0,08 & Treonina & 0,14 & Magnésio & 4215 \\
\hline Ácido a-linoleico & 0,01 & Valina & 0,14 & Alumínio & 127 \\
\hline Ácido araquidônico & 0,01 & Metionina & 0,03 & & \\
\hline Gordura insaturada & 0,61 & Isoleucina & 0,11 & & \\
\hline Gordura saturada & 0,66 & Leucina & 0,20 & & \\
\hline Ômega 3 & 0,02 & Fenilalanina & 0,12 & & \\
\hline Ômega 6 & 0,24 & Lisina & 0,09 & & \\
\hline Ômega 9 & 0,30 & AAs totais & 2,06 & & \\
\hline
\end{tabular}

${ }^{1}$ Valores expressos na matéria natural do material como recebido (77\% MS)

${ }^{2}$ Valores expressos na matéria seca.

O ensaio de metabolismo foi realizado em um período de cinco dias (15ํㅡ ao $20^{\circ}$ dia de idade), através do método de coleta total de excretas. As excretas foram coletadas em bandejas metálicas cobertas com material plástico duas vezes ao dia, às 08:00 e às 16:00 horas, de modo a evitar fermentação fecal e contaminações. As excretas coletadas foram acondicionadas em sacos plásticos identificados, pesadas e congeladas. Amostras das rações experimentais foram acondicionadas em potes fechados e identificados e sendo congelada para posterior análise centesimal. Para as análises, as excretas foram descongeladas à temperatura ambiente e homogeneizadas para a retirada de uma alíquota por unidade experimental. As amostras foram secas em estufa de ventilação forçada a $55^{\circ} \mathrm{C}$ por 72 horas. Em seguida foram moídas em micromoinho de facas (peneira de $1 \mathrm{~mm}$ ) e encaminhadas para análises de matéria seca (MS), nitrogênio $(\mathrm{N})$ e energia bruta $(E B)$, matéria mineral $(M M)$ e fósforo $(P)$.

Com base nos dados de consumo de ração, excreta total, análises de MS, N, EB, MM e P das rações e das excretas, foram determinados os coeficientes de digestibilidade aparente da matéria seca (CDMS), proteína bruta (CDPB), energia bruta (CDEB), e os coeficientes de retenção de matéria mineral (CRMM) e fósforo (CRP). A energia metabolizável aparente (EMA) e energia metabolizável aparente corrigida para o 
balanço de nitrogênio (EMAn), que foram calculados através das fórmulas propostas por MATTERSON et al. (1965).

Tabela 2. Composição percentual da ração referência.

Table 2. Percentage composition of the reference diet.

\begin{tabular}{|c|c|}
\hline Ingredientes & Composição (\%) \\
\hline Farelo de soja & 44,356 \\
\hline Milho & 42,710 \\
\hline Óleo de soja & 6,719 \\
\hline Inerte $^{3}$ & 2,500 \\
\hline Fosfato bicálcico & 1,625 \\
\hline Sal comum & 0,516 \\
\hline DL-Metionina & 0,336 \\
\hline Suplemento vitamínico ${ }^{1}$ & 0,100 \\
\hline Suplemento mineral ${ }^{2}$ & 0,100 \\
\hline Cloreto de colina & 0,083 \\
\hline L-Lisina HCL & 0,073 \\
\hline L-Treonina & 0,050 \\
\hline Antioxidante $(\mathrm{BHT})$ & 0,010 \\
\hline Nutrientes & Composição calculada \\
\hline Ácido linoleico (\%) & 4,741 \\
\hline Arginina digestível (\%) & 1,508 \\
\hline Cálcio (\%) & 0,878 \\
\hline Cloro (\%) & 0,368 \\
\hline Energia metabolizável (kcal/kg) & 3.050 \\
\hline Fósforo disponível (\%) & 0,419 \\
\hline Lisina digestível (\%) & 1,256 \\
\hline Metionina + Cistina digestíveis (\%) & 1,024 \\
\hline Metionina digestível (\%) & 0,633 \\
\hline Potássio (\%) & 0,948 \\
\hline Proteína bruta (\%) & 23,31 \\
\hline Sódio (\%) & 0,218 \\
\hline Treonina digestível (\%) & 0,831 \\
\hline Triptofano digestível (\%) & 0,274 \\
\hline
\end{tabular}

Ferro (min) $60 \mathrm{~g} / \mathrm{kg}$; cobre (min) $13 \mathrm{~g} / \mathrm{kg}$; manganês (min) $120 \mathrm{~g} / \mathrm{kg}$; zinco (min) $100 \mathrm{~g} / \mathrm{kg}$; iodo (min) 2.500 mg/kg; selênio (min) $500 \mathrm{mg} / \mathrm{kg} .{ }^{2}$ Vitamina A (min) $7.500 .000 \mathrm{Ul} / \mathrm{kg}$; vitamina D3 (min) 2.500.000 Ul/kg; vitamina E (min) 1.200 $\mathrm{mg} / \mathrm{kg}$; vitamina K3 (min) $1.200 \mathrm{mg} / \mathrm{kg}$; tiamina (min) $1.500 \mathrm{mg} / \mathrm{kg}$; riboflavina (min) $5.500 \mathrm{mg} / \mathrm{kg}$; piridoxina (min) 2000 $\mathrm{mg} / \mathrm{kg}$; vitamina B12 (min) $12.000 \mathrm{mcg} / \mathrm{kg}$; niacina 35g/kg; panteonato de cálcio (min) $10 \mathrm{~g} / \mathrm{kg}$; biotina (min) $67 \mathrm{mg} / \mathrm{kg}$. ${ }^{3}$ Inerte - Caulim.

Aos 21 dias de idade uma ave por repetição foi selecionada de acordo com o peso médio e abatida para coleta dos tibiotarsos para a avaliação de qualidade óssea. Os ossos foram congelados e identificados, sendo posteriormente descongelados e processados de acordo com metodologia descrita por BRUNO (2002). Após a retirada do tecido muscular dos tibiotarsos esquerdos, foram realizadas as análises de Índice de Seedor (SEEDOR et al. 1991), resistência óssea (FAITARONE et al. 2012) e cinzas ósseas.

Para obter-se o índice de Seedor, os tibiotarsos esquerdos foram pesados em balança semianalítica e posteriormente, mensurado o comprimento deles com paquímetro analógico. O índice de Seedor é calculado a partir da razão entro o peso e o comprimento ósseo, sendo utilizado como um indicativo da densidade óssea, quanto maior o índice de Seedor maior a densidade óssea.

Para a avaliação de resistência óssea, as tíbias esquerdas foram apoiadas em um suporte de madeira com um vão-livre de $2 \mathrm{~cm}$ e acoplado ao equipamento Texture Analyser TA.XT Plus, com a utilização da sonda Blade Set HDP/BS. A velocidade de pré-teste foi de 2,0 mm/segundo, a velocidade de teste de $1,0 \mathrm{~mm} / \mathrm{segundo}$ e a velocidade de pós-teste de $4,0 \mathrm{~mm} / \mathrm{segundo}$. Um software específico foi utilizado para registrar a força necessária empregada para o rompimento total dos ossos em Newton, sendo esse valor transformado em quilograma-força (kgf). 
Após a realização do teste de resistência óssea, os ossos partidos foram pesados, mantidos em estufa a $105{ }^{\circ} \mathrm{C}$ por 12 horas e posteriormente levado ao forno tipo mufla a $580{ }^{\circ} \mathrm{C}$ por quatro horas e pesados novamente, para cálculo de porcentagem de cinzas.

Os resultados foram submetidos à ANOVA $(\alpha=0,05)$ utilizando-se o pacote estatístico SISVAR. A análise de variância foi realizada considerando o delineamento experimental inteiramente casualizado, sendo descrito segundo o modelo estatístico: $Y i k=\mu+T i+\varepsilon i j$, sendo: $Y i j=$ variáveis dependentes estudadas; $\mu=$ média geral; $\mathrm{Ti}=$ efeito do nível de inclusão da macroalga Kappaphycus alvarezii, sendo i =0; 0,5; 1,0; 1,$5 ; 2,0 \% ; \varepsilon i j$ = erro aleatório associado a cada observação.

Quando significativa a ANOVA, os dados foram submetidos à análise de regressão, ao nível de $5 \%$ de probabilidade.

\section{RESULTADOS E DISCUSSÃO}

Em relação aos resultados de energia metabolizável aparente $\left(Y=-125,22 x+3641, R^{2}=0,96\right)$ e energia metabolizável aparente corrigida para o balanço do nitrogênio $\left(Y=-122,75 x+3387, R^{2}=0,91\right)$ (Tabela 3) houve diminuição linear do valor energético da ração com o aumento da inclusão da Kappaphycus alvarezii. Os coeficientes de metabolizabilidade da matéria seca $\left(Y=2,47 x^{2}-6,76 x+72,52\right.$, $\left.R^{2}=0,81\right)$, da proteína bruta $\left(Y=3,31 x^{2}-9,59+69,99, R^{2}=0,79\right)$ e da energia bruta $\left(Y=1,31 x^{2}-4,99+\right.$ $\left.78,72, R^{2}=0,94\right)$, apresentaram efeito quadrático decrescente com o aumento do nível de inclusão da macroalga avaliada. Os valores de inclusão da macroalga $K$. alvarezii de 1,37\%; $1,45 \%$ e de $1,91 \%$ resultariam nos menores valores de coeficientes de metabolizabilidade aparente da matéria seca, da proteína bruta e da energia bruta, 67,89\%,63,06\% e 73,95\%, respectivamente.

Tabela 3. Resultados de energia metabolizável aparente (EMA), energia metabolizável aparente corrigida para o balanço de nitrogênio (EMAn) e os coeficientes de metabolizabilidade aparente da matéria seca (CDMS), proteína bruta (CDPB), energia bruta (CDEB), e os coeficientes de retenção de matéria mineral (CRMM) e fósforo (CRP) de rações contendo níveis crescentes de inclusão da macroalga $K$. alvarezii.

Table 3. Results for apparent metabolizable energy (ME); nitrogen-corrected apparent metabolizable energy (MEn) and the coefficients of digestibility for dry matter (CDDM), crude protein (CDCP), crude energy (CDCE); and the coefficients of retention for mineral matter (CRMM) and phosphorus $(C R P)$ in diets incorporating increasing levels of the macroalgae $K$. alvarezii.

\begin{tabular}{lcccccrrr}
\hline \multicolumn{1}{c}{ Variáveis } & \multicolumn{9}{c}{ Inclusão da macroalga K. alvarezii (\%) } & CV (\%) & p-valor & Efeito \\
\hline & 0 & 0,5 & 1,0 & 1,5 & \multicolumn{1}{c}{2,0} & & & \\
\hline EMA $^{1}(\mathrm{kcal} / \mathrm{kg})$ & 3.644 & 3.588 & 3.516 & 3.421 & 3.414 & 2,64 & 0,0025 & $\mathrm{~L}$ \\
EMAn $^{1}(\mathrm{kcal} / \mathrm{kg})$ & 3.377 & 3.362 & 3.254 & 3.162 & 3.170 & 2,59 & 0,0009 & $\mathrm{~L}$ \\
CDMS (\%) & 72,94 & 68,52 & 69,39 & 67,63 & 68,85 & 2,96 & 0,0061 & $\mathrm{Q}$ \\
CDPB (\%) & 70,89 & 63,78 & 65,09 & 63,49 & 63,63 & 3,96 & 0,008 & $\mathrm{Q}$ \\
CDEB (\%) & 78,88 & 76,01 & 75,74 & 73,77 & 74,05 & 2,33 & 0,0014 & $\mathrm{Q}$ \\
CRMM (\%) & 39,38 & 36,52 & 34,77 & 34,25 & 36,92 & 10,40 & 0,2587 & $\mathrm{NS}$ \\
CRP (\%) & 61,96 & 58,14 & 59,35 & 56,04 & 57,55 & 5,47 & 0,0845 & $\mathrm{NS}$ \\
\hline
\end{tabular}

${ }^{1}$ Valores expressos na base seca.

A composição da alga em FDN e FDA podem ter aumentado o teor de polissacarídeos não amiláceos (PNAs) nas dietas. Os PNAs são considerados fatores antinutricionais para monogástricos, devido a sua baixa digestão por estes animais e a elevada capacidade de ligar-se a grandes quantidades de água no lúmen intestinal, resultando num aumento da viscosidade do conteúdo intestinal, diminuindo a taxa de passagem dos alimentos ao longo do trato digestivo, dificultando a ação das enzimas endógenas e interferindo na difusão ou transporte dos nutrientes (BRITO et al. 2008). Este fato poderia explicar a redução da digestibilidade de nutrientes e do valor energético das dietas.

Além disso, a presença de carragena em altos níveis na Kappaphycus alvarezii (33 a 43\%) (SYAMSUDDIN et al. 2019), também podem afetar a digestibilidade de nutrientes. A carragena é um grupo de polissacarídeos sulfatados formadores de gel e viscosificantes (NECAS \& BARTOSIKOVA 2013). Em estudos com ratos, HARMUTH-HOENE \& SCHWERDTFEGER (1979) relataram que a inclusão de $10 \%$ de carragena foi capaz de reduzir a digestibilidade da proteína e da matéria seca. Esta diminuição está relacionada a interação entre polissacarídeos ácidos e os grupos ativos de quimotripsina e tripsina.

Quanto aos coeficientes de retenção da matéria mineral e do fósforo não foram observados efeitos 
dos valores de inclusão da macroalga Kappaphycus alvarezii. O caulim é composto por silicatos que não são absorvidos pelos animais, sendo, portanto, classificado como inerte. Assim a fração de matéria mineral passível de absorção ou com valor como nutriente teoricamente aumentou com a inclusão da macroalga, entretanto, isso não se refletiu no aumento dos coeficientes de retenção da matéria mineral das rações estudadas.

Quanto aos resultados de desempenho de frangos de corte no período de 1 a 21 dias (Tabela 4), não foram observadas diferenças significativas $(p>0,05)$ nas variáveis estudadas. Portanto, a inclusão da alga em até $2 \%$ em dietas para frangos de corte não prejudicou o desenvolvimento das aves, apesar da redução da digestibilidade da dieta. Esses resultados são semelhantes aos descritos por QADRI et al. (2019a), que avaliaram a inclusão de até $1,5 \%$ da macroalga Kappaphycus alvarezii em rações de frangos de corte no período de 1 a 21 dias e relataram que os tratamentos não afetaram o desempenho dos frangos.

Tabela 4. Desempenho zootécnico de frangos de corte de 1 a 21 dias alimentados com rações contendo níveis crescentes de inclusão da macroalga $K$. alvarezii.

Table 4. Zootechnical performance of broilers fed a diet containing increasing levels of the macroalgae $K$. alvarezii from 1 to 21 days of age.

\begin{tabular}{|c|c|c|c|c|c|c|c|}
\hline \multirow{2}{*}{ Variáveis } & \multicolumn{5}{|c|}{ Inclusão da macroalga K. alvarezii (\%) } & \multirow{2}{*}{ CV (\%) } & \multirow{2}{*}{ p-valor } \\
\hline & 0 & 0,5 & 1,0 & 1,5 & 2,0 & & \\
\hline PV $(g)$ & 1004 & 1041 & 1003 & 987 & 996 & 4,62 & 0,4324 \\
\hline GP $(g)$ & 967 & 1004 & 966 & 949 & 959 & 4,80 & 0,4311 \\
\hline CR (g) & 1173 & 1186 & 1126 & 1134 & 1136 & 4,78 & 0,3502 \\
\hline $\mathrm{CA}(\mathrm{g} / \mathrm{g})$ & 1,21 & 1,18 & 1,17 & 1,19 & 1,18 & 3,23 & 0,3967 \\
\hline
\end{tabular}

$\overline{\mathrm{CV}}=$ Coeficiente de Variação, $\mathrm{PV}=$ Peso vivo, $\mathrm{GP}=$ Ganho de peso, $\mathrm{CR}=$ Consumo de ração, $\mathrm{CA}=$ Conversão alimentar, VB = Viabilidade de criação.

QADRI et al. (2019a) conduziram o estudo até os 42 dias de idade, e observaram que a inclusão de até $1,25 \%$ da macroalga refletiu em maior ganho de peso. Além disso os autores observaram que houve uma melhora da resposta imune das aves. Aves jovens apresentam menor capacidade digestiva e maior sensibilidade aos fatores antinutricionais do que as aves mais velhas (DILELIS et al. 2019). KRÁS et al. (2013), demonstraram que à medida que as aves crescem, melhoram também a digestibilidade da fibra. Estes fatos podem ajudar a explicar os resultados positivos obtidos em aves mais velhas. Em relação a digestibilidade, não é do conhecimento destes autores trabalhos que determinaram esta variável com o uso de Kappaphycus alvarezii.

Conforme a análise dos dados dos parâmetros ósseos avaliados (Tabela 5), a inclusão da macroalga na ração não influenciou significativamente $(p>0,05)$ as variáveis Índice de Seedor, resistência óssea e cinzas ósseas, evidenciando que a adição de Kappaphycus alvarezii às rações não interferiu na dinâmica de deposição e formação da matriz óssea. Na literatura consultada não foram encontrados resultados de retenção de minerais, seja com a Kappaphycus alvarezii ou outra macroalga, que permitisse a comparação com os dados obtidos neste experimento.

Tabela 5. Índice de Seedor e resistência e cinzas ósseas de frangos de corte aos 21 dias de idade alimentados com rações contendo níveis crescentes de inclusão da macroalga $K$. alvarezii.

Table 5. Seedor index and bone strength and ash for 21-day-old broiler chickens fed a diet containing increasing levels of the macroalgae $K$. alvarezii.

\begin{tabular}{lcccccrc}
\hline \multirow{2}{*}{ Variáveis } & \multicolumn{9}{c}{ Inclusão da macroalga $K$. alvarezii (\%) } & \multirow{2}{*}{ CV (\%) $)^{*}$ p-valor } \\
\cline { 2 - 6 } & 0 & 0,5 & 1,0 & 1,5 & 2,0 & & \\
\hline Índice de Seedor & $80,55^{2}$ & 81,45 & 78,91 & 74,93 & 74,28 & 7,44 & 0,1473 \\
Resistência óssea (kgf) & 27,94 & 25,41 & 25,85 & 23,69 & 24,11 & 19,81 & 0,6226 \\
Cinzas ósseas (\%) & 51,42 & 50,62 & 49,79 & 50,98 & 50,92 & 2,23 & 0,1786 \\
\hline
\end{tabular}

${ }^{1} \mathrm{CV}=$ Coeficiente de Variação.

Os benefícios da utilização de algas na dieta de animais, além do valor nutricional, podem estar associados à presença de compostos bioativos, pois estes compostos podem exercer funções antifúngicas, 
antimicrobianas, imunoestimulantes e antioxidantes (CORNISH \& GARBARY 2010, IJI \& KADAM 2013, PUSHPARAJ et al. 2014, QADRI et al. 2019a). QADRI et al. (2019b) relatam que a inclusão de até 1,5\% da Kappaphycus em dietas de frangos melhoraram parâmetros antioxidantes da carne, bem como reduziu a contagem de microrganismos patógenos.

Apesar da utilização da macroalga em até $2 \%$ ter reduzido a digestibilidade da ração, não influenciou o desempenho dos frangos de corte. O esperado com a redução da digestibilidade seria a piora dos parâmetros de desempenho. Uma possibilidade é que os efeitos relatados ao uso dessa alga como aditivo tenham sido capazes de manter o desempenho das aves, mesmo com a piora da digestibilidade.

Alguns trabalhos têm buscado qualificar a presença de compostos bioativos em extratos de Kappaphycus alvarezii. PRABHA et al. (2013) qualificaram a presença de diferentes compostos fitoquímicos nos diferentes extratos desta macroalga, dentre eles: alcaloides, taninos, terpenoides, flavonoides, saponinas, compostos fenólicos, entre outros. Não foi possível estudar esses aspectos no presente trabalho, sendo necessário a realização de experimentos que esclareçam o potencial do uso da Kappaphycus alvarezii como aditivo nas rações.

\section{CONCLUSÃO}

A inclusão da macroalga resultou na redução dos valores energéticos e dos coeficientes de digestibilidade da matéria seca, da proteína bruta e da energia bruta das rações, sem, entretanto, influenciar negativamente os parâmetros de desempenho, de qualidade óssea e os coeficientes de retenção de matéria mineral e do fósforo dos frangos de corte. A alga Kappaphycus alvarezii apresenta potencial como aditivo nas rações de frangos de corte, podendo ser incluída em até $2 \%$ sem prejuízo no desempenho das aves.

\section{AGRADECIMENTOS}

O presente trabalho foi realizado com apoio da Coordenação de Aperfeiçoamento de Pessoal de Nível Superior - Brasil (CAPES) - Código de Financiamento 001.

\section{REFERÊNCIAS}

ABUDABOS AM et al. 2013. Nutritional Value of Green Seaweed (Ulva Lactuca) for Broiler Chickens. Italian Journal of Animal Science 12: e.28.

AOAC. 1995. Association of Official Analytical Chemistry. Official methods of analysis. 16.ed. Arlington: AOAC International.

BRITO MS et al. 2008. Polissacarídeos não amiláceos na nutrição de monogástricos - Revisão. Acta Veterinaria Brasilica 2: 111-117.

BRUNO LDG. 2002. Desenvolvimento ósseo em frangos: influência da restrição alimentar e da temperatura ambiente. Tese (Doutorado em Zootecnia). Piracicaba: UNESP. 77p.

CORNISH ML \& GARBARY DJ. 2010. Antioxidants from macroalgae: potential applications in human health and nutrition. Algae 25: 155-171.

DILELIS F et al. 2019. Metabolizable energy of rice bran, cottonseed meal and wheat bran for slow-growing broilers at two ages. Ciência Animal Brasileira 20: e46537.

EL GAMAL AA. 2012. Biological importance of marine algae. In: Se-Kwon Kim (Ed.) Handbook of marine macroalgae: biotechnology and applied phycology. Nova Jersey: John Wiley \& Sons.

FAITARONE ABG et al. 2012. Qualidade óssea de poedeiras comerciais leves alimentadas com rações suplementadas com diferentes óleos vegetais. Veterinária e Zootecnia 19: 356-365.

FAO. 2012. The state of world fisheries and aquaculture. Rome: FAO. 210p.

HARMUTH-HOENE A \& SCHWERDTFEGER E. 1979. Effect of Indigestible Polysaccharides on Protein Digestibility and Nitrogen Retention in Growing Rats. Annals of Nutrition and Metabolism 23: 399-407.

IJI PA \& KADAM MM. 2013. Prebiotic properties of algae and algae-supplemented products A2 In: DOMíNGUEZ H. (Ed.) Functional Ingredients from Algae for Foods and Nutraceuticals. Cambridge: Woodhead Publishing. p.658-670.

INSTITUTO ADOLFO LUTZ. 2008. Métodos físico-químicos para análise de alimentos. São Paulo: Instituto Adolfo Lutz. 1020p.

KRÁS RV et al. 2013. Effect of dietary fiber, genetic strain and age on the digestive metabolism of broiler chickens. Brazilian Journal of Poultry Science 15: 83-90.

KUMAR KS et al. 2015. Seasonal variation in nutritional composition of Kappaphycus alvarezii (Doty) Doty-an edible seaweed. Journal of Food Science and Technology 52: 2751-2760.

MATTERSON LS et al. 1965. The metabolizable energy of feed ingredients for chickens. Storrs: Agricultural Experiment Station Research Report 7: 11.

MELO TV et al. 2008. Calidad del huevo de codornices utilizando harina de algas marinas y fosfato monoamónico. Archivos de Zootecnia 57: 313-319.

NECAS J \& BARTOSIKOVA L. 2013. Carrageenan: a review. Veterinarni Medicina 58: 187-205. 
PRABHA V et al. 2013. Analysis of Bioactive compounds and Antimicrobial activity of Marine algae Kappaphycus alvarezii using Three Solvent Extracts. International Journal of Pharmacy and Pharmaceutical Sciences and Research 4: 306-310.

PUSHPARAJ A et al. 2014. An Antibacterial activity of the Green Seaweed Caulerpha Sertularioides using Five Different Solvents. International Journal of ChemTech Research 6: 1-5.

QADRI SSN et al. 2019a. Production performance, immune response and carcass traits of broiler chickens fed diet incorporated with Kappaphycus alvarezii. Journal of Applied Phycology 31: 753-760.

QADRI SSN et al. 2019b. Physico-biochemical and microbial characteristics of broiler chicken meat fed diet incorporated with Kappaphycus alvarezii. Journal of Applied Phycology 31: 3949-3955.

ROSTAGNO HS et al. 2017. Tabelas brasileiras para aves e suínos: composição de alimentos e exigências nutricionais de aves e suínos. 4.ed. Viçosa: UFV. 488p.

SEEDOR JG et al. 1991. The biophosphonate alendronate (MK-217) inhibits boné loss due to ovariectomy in ratas. Journal of Bone and Mineral Research 6: 339-346.

SYAMSUDDIN R et al. 2019. Weight gain and carrageenan content of Kappaphycus alvarezii (Rhodophyta, Solierisceae) polycultured with Sargassum polycystum (Paeophyta, Sargassaceae). In: IOP Conf. Series: Earth and Environmental Science. p.253.

VAN SOEST PJ et al. 1991. Methods for dietary fiber, neutral detergent fiber, and nonstarch polysaccharides in relation to animal nutrition. Journal of Dairy Science 74: 3583-3597.

XIREN GK \& AMINAH A. 2017. Kappaphycus alvarezii found in the waters of Langkawi and Sabah, Malaysia. International Food Research Journal 24: 1255-1260. 